\title{
Service Item
}

National Cancer Institute

\section{Source}

National Cancer Institute. Service Item. NCI Thesaurus. Code C88187.

A distinct item or unit of work available by fee for service purchase. 